\title{
The predictive value of plasminogen activator inhibitor-1, fibrinogen, and D-dimer for deep venous thrombosis following surgery for traumatic lower limb fracture
}

\author{
Jie Cheng ${ }^{1}$, Zhibin $\mathrm{Fu}^{1}$, Jianxin $\mathrm{Zhu}^{2}$, Lin Zhou ${ }^{1}$, Weijun Song ${ }^{1}$ \\ ${ }^{1}$ Department of Lower Limbs Ward, Sport Hospital Affiliated to CDSU, Chengdu, China; ${ }^{2}$ Department of Orthopaedic, Sichuan Academy of \\ Medical Sciences, Sichuan Provincial People's Hospital, Chengdu, China \\ Contributions: (I) Conception and design: J Cheng, Z Fu; (II) Administrative support: J Cheng, L Zhou; (III) Provision of study materials or patients: \\ J Cheng, Z Fu, J Zhu, W Song; (IV) Collection and assembly of data: All authors; (V) Data analysis and interpretation: J Cheng, J Zhu, L Zhou, W \\ Song; (VI) Manuscript writing: All authors; (VII) Final approval of manuscript: All authors. \\ Correspondence to: Jie Cheng. Department of Lower Limbs Ward, Sport Hospital Affiliated to CDSU, No. 251, Wuhouci Street, Chengdu, China. \\ Email: chengjiecdty@163.com.
}

Background: Deep venous thrombosis (DVT) is a common postoperative complication in patients with lower limb fractures. This study aims to investigate the predictive value of plasminogen activator inhibitor-1 (PAI-1), fibrinogen (FIB), and D-dimer (D-D) for DVT following lower limb traumatic fracture surgery and to investigate risk factors for DVT.

Methods: Clinical data of 63 patients who underwent lower limb traumatic fracture surgery in our hospital from September 2018 to March 2019 were retrospectively analyzed. Patients were divided into a DVT group and a non-DVT group. The differences in the levels of plasminogen activator inhibitor-1 (PAI-1), fibrinogen (FIB), and D-dimer (D-D) were compared, and a receiver operating characteristic (ROC) curve was used to analyze their predictive value for DVT following surgery for lower limb traumatic fracture. Multiple logistic regression analysis was used to analyze the risk factors of DVT.

Results: The levels of PAI-1, FIB, and D-D in the DVT group were higher on the third day after surgery compared to the pre-surgical levels, and were also higher than those in the non-DVT group $(\mathrm{P}<0.05)$. The area under the ROC curve indicated that the predictive values of PAI-1, FIB, D-D, and the combination of these three indicators for DVT were $0.792,0.429,0.966$, and 0.992, respectively. Patients with preexisting factors including a $\mathrm{BMI} \geq 24 \mathrm{~kg} / \mathrm{m}^{2}$, a history of diabetes, postoperative infection, an abnormal white blood cell count, an abnormal average thrombocytocrit, and abnormal levels of PAI-1, FIB, and D-D had a higher incidence of DVT following surgery compared to patients without these factors $(\mathrm{P}<0.05)$. The results of the multivariate logistic regression model analysis showed that the presence of postoperative infection, abnormal white blood cell count, abnormal mean platelet volume, and abnormal levels of PAI-1, FIB, and D-D were independent risk factors affecting postoperative DVT in patients with lower limb fractures $(\mathrm{P}<0.05)$.

Conclusions: The levels of PAI-1, FIB, and D-D were significantly increased in patients with DVT following surgery for lower limb fractures. Therefore, early monitoring of PAI-1, FIB, and D-D levels, and coagulation function is a good predictive indicator of postoperative thrombosis.

Keywords: Plasminogen activator inhibitor-1 (PAI-1); fibrinogen (FIB); D-dimer (D-D); traumatic fracture of lower limb; deep venous thrombosis (DVT)

Submitted Jul 28, 2020. Accepted for publication Sep 08, 2020.

doi: 10.21037/apm-20-1604

View this article at: http://dx.doi.org/10.21037/apm-20-1604 


\section{Introduction}

Deep venous thrombosis (DVT) refers to the formation of a blood clot within a deep vein, resulting in vascular stenosis or occlusion (1). DVT is a common complication after surgery of lower limb fractures. However, studies have reported that only $10-17 \%$ of DVT patients present with obvious clinical symptoms, such as swelling of the lower limbs, local deep tenderness and dorsiflexion pain (2), which has led to high rates of missed diagnosis and misdiagnosis of DVT in the lower limbs. Therefore, early diagnosis and intervention for high-risk patients has considerable clinical significance.

At present, the commonly used methods for the diagnosis of DVT include intravenous color ultrasound, venography, and other imaging detection methods. Although these techniques show certain specificity and sensitivity, they cannot be used for the prediction of DVT occurrence. At present, there are many studies in China on the prognostic factors of patients with DVT after the operation of lower limb venous fracture, but there are few researches on the relevant prediction methods. Therefore, focusing on the perspective of molecular biological mechanism, this study aims to provide novel ideas for clinical treatment (3). Smooth muscle cells are critical structural components of the peripheral wall of blood vessels. Their phenotype is often changed in the process of vascular stability, where plasminogen activator inhibitor-1 (PAI-1) plays a key role in regulating their function (4). Fibrinogen (FIB) is a glycoprotein synthesized and secreted by liver cells, and is involved in coagulation and hemostasis. PAI-1 and D-Dimer (D-D) are both clinically recognized biochemical indicators of thrombotic diseases (5). In this study, the pre- and postoperative levels of PAI-1, FIB, and D-D were measured in patients with lower limb traumatic fractures to explore their predictive value for DVT and identify the risk factors for DVT. We present the following article in accordance with the MDAR reporting checklist (available at http://dx.doi.org/10.21037/apm-20-1604).

\section{Methods}

\section{General information}

Clinical data of 63 patients who underwent surgical treatment in our hospital for lower limb trauma fractures from September 2018 to March 2019 were retrospectively analyzed. Patients were divided into a DVT group and a non-DVT group depending on whether DVT occurred within 2 weeks after surgery.

Inclusion criteria: (I) patients in the DVT group were required to meet the diagnostic criteria for DVT (6), namely that more than $95 \%$ of the veins could not be compressed or that there was no blood flow signal in the venous cavity as measured using ultrasound probe compression observation or extrusion of distal limbs; (II) patients were diagnosed with lower limb fractures and received surgical treatment; (III) the clinical data was complete with no missing information; (IV) no lower limb DVT was detected by color Doppler ultrasound prior to surgery.

Exclusion criteria: (I) patients with multiple fractures throughout the body; (II) patients with malignant tumors; (III) patients with DVT in the preoperative examination; (IV) patients with a history of pulmonary embolism or lower limb DVT; (V) patients with complications such as cardiovascular, respiratory, or other serious diseases. All procedures performed in this study involving human participants were in accordance with the Declaration of Helsinki (as revised in 2013). This study was approved by Sport Hospital Affiliated to CDSU (No. 2018-12). And all the patients were signed the informed consent.

\section{Data collection and detection of PAI-1, FIB, and D-D levels}

General and clinical data of all patients were collected including gender, age, body mass index (BMI), lower limb fracture site, diabetes status, preoperative platelet count, white blood cell count, preoperative coagulation, preoperative liver and kidney function, operation time, the amount of blood loss during the operation, whether the patient had received intraoperative blood transfusion, whether the patient had a postoperative infection, the amount of postoperative blood loss, postoperative anticoagulant use, and the levels of PAI-1, FIB, and D-D. The levels of PAI-1, FIB, and D-D were compared between the two groups.

\section{Observation indicators}

Fasting venous blood $(5 \mathrm{~mL})$ was collected before and on the third day after surgery, and injected into a $0.2 \mathrm{~mL}$ test tube pretreated with $0.25 \%$ sodium citrate anticoagulant, centrifuged (3,000 r/min, $10 \mathrm{~min})$, and then aliquoted. The concentrations of PAI-1, FIB, and D-D were detected using enzyme-linked immunosorbent assay (ELISA). The levels of PAI-1 and FIB were detected using the original matching detection kit (STAGO, France), and the D-D level was detected using the immunoturbidimetric method. The NycoCard-READER II multifunctional full quantitative 
Table 1 Significance and assignment of factors



PAI-1, plasminogen activator inhibitor-1; FIB, fibrinogen; D-D, D-dimer; BMI, body mass index.

detection instrument (Nycomed, Norway) and the matching test kit were operated in strict accordance with the operating instructions. The normal range of PAI- 1 was $2.5-80 \mathrm{ng} / \mathrm{mL}$; the normal range of FIB was $2.0-4.0 \mathrm{~g} / \mathrm{L}$; the normal range of D-D was $0-400 \mu \mathrm{g} / \mathrm{L}$.

\section{Statistical methods}

Results were analyzed using SPSS 20.0 statistical software (IBM, USA). The measurement data of clinical indexes was expressed as $(\bar{x} \pm S)$ and analyzed using the $\mathrm{F} / t$ test. The count data was expressed as a percentage (\%) and analyzed using the Chi-square $\left(\chi^{2}\right)$ test. The receiver operating characteristic (ROC) curve was used to assess the predictive value of PAI-1, FIB, and D-D levels in patients with DVT following lower limb fracture surgery. Logistic regression analysis was used for analyzing the related risk factors of DVT. The variables and assignments are shown in Table 1, with $\alpha=0.05$ as the test standard.

\section{Results}

\section{Comparison of the levels of PAI-1, FIB, and D-D between} the two groups

There were no significant differences in the preoperative levels of PAI-1, FIB, and D-D between the two groups $(\mathrm{P}>0.05)$. The levels of PAI-1, FIB, and D-D in the DVT group on the third day after surgery were higher than the pre-surgical levels, and were also higher than those in the non-DVT group $(\mathrm{P}<0.05$, Table 2).

\section{The predictive value of PAI-1, FIB, and D-D for postoperative DVT in patients with lower limb fractures}

The ROC curve was used to analyze the predictive value of PAI-1, FIB, D-D, and the combination of these three indicators for DVT following lower limb fracture surgery. The area under the curve of each index was $0.792,0.429$, 0.966 , and 0.992 , respectively. The detection of the combination of these three indicators exhibited the best predictive value (Figure 1).

\section{Single-factor analysis of postoperative DVT in patients with lower limb fractures}

There were 30 patients in the DVT group (17 males, and 13 females, aged 28-70 years old, with an average age of $45.93 \pm 5.39$ years) and 33 patients in the non-DVT group (16 males and 17 females, aged 28-72 years old, with an average age $46.71 \pm 5.21$ years old). There were no significant 
Table 2 Comparison of PAI-1, FIB, and D-D levels $(\bar{x} \pm s)$



*, compared with presurgical levels, $\mathrm{P}<0.05$. PAI-1, plasminogen activator inhibitor-1; FIB, fibrinogen; D-D, D-dimer.

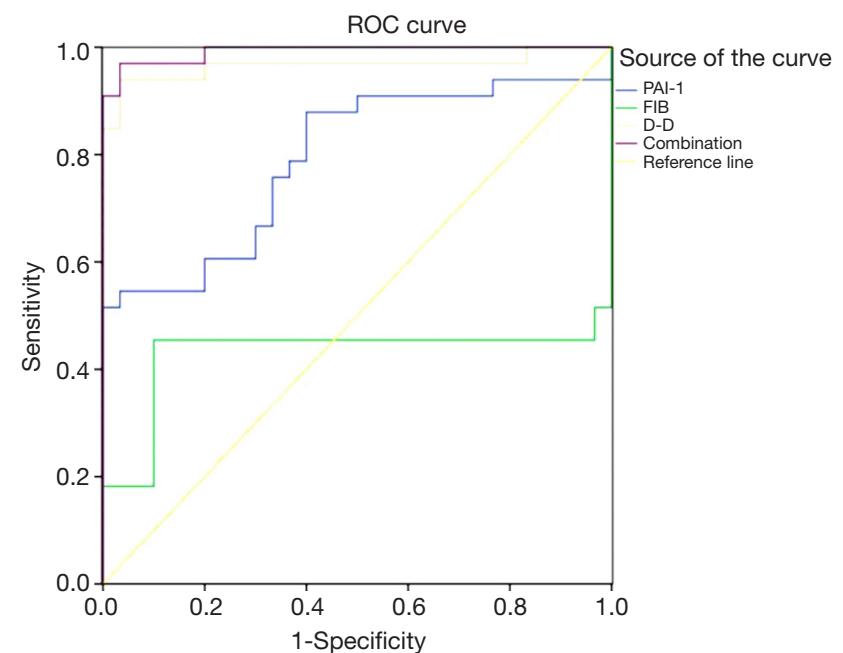

Figure 1 Predictive value of PAI-1, FIB, and D-D for postoperative DVT in patients with lower limb fractures. PAI-1, plasminogen activator inhibitor-1; FIB, fibrinogen; D-D, D-dimer; DV'T, deep venous thrombosis.

differences in the general data between the two groups (such as age and gender), and thus they were comparable $(\mathrm{P}>0.05$, Table 3).

The risk factors and assignments of DVT following lower limb fracture surgery are shown in Table 1. According to the results of the single-factor logistic regression analysis, patients with a BMI $\geq 24 \mathrm{~kg} / \mathrm{m}^{2}$, a history of diabetes, postoperative infection, abnormal white blood cell count, abnormal mean platelet volume, and abnormal levels of PAI-1, FIB and D-D had a higher postoperative DVT rate than those without these factors $(\mathrm{P}<0.05$, Table 3$)$.

\section{Multivariate analysis of risk factors affecting postoperative DVT in patients with lower limb fractures}

The results of the unconditional multivariate logistic regression model analysis showed that factors including the presence of postoperative infection, abnormal white blood cell count, abnormal mean platelet volume, and abnormal levels of PAI-1, FIB, and D-D were independent risk factors for postoperative DVT in patients with lower limb fractures $(\mathrm{P}<0.05$, Table 4).

\section{Discussion}

DVT is an abnormal coagulation of blood in deep veins, resulting in vascular stenosis or occlusion (1). DVT is a common postoperative complication following lower limb fracture surgery, and affects venous return (7). Thrombosis often occurs at the state of braking (especially during orthopedic surgery, which may be related to the following factors: (I) reposition and fixation during surgery may cause damage to the veins around the fracture, resulting in hemodynamic changes; (II) preoperative fasting and water deprivation may cause the blood to be concentrated, which may in turn increase the risk of DVT; (III) early postoperative pain and local edema may restrict limb activity and slow the flow of blood; (IV) the postoperative stress response may increase platelet cohesion $(8,9)$. Therefore, to improve quality of life, enhancing the postoperative risk assessment of DVT in fracture patients is crucial, in addition to early diagnosis and treatment (10).

The fibrinolytic system is a critical anticoagulant 
Table 3 Single-factor analysis of postoperative DVT in patients with lower limb fractures

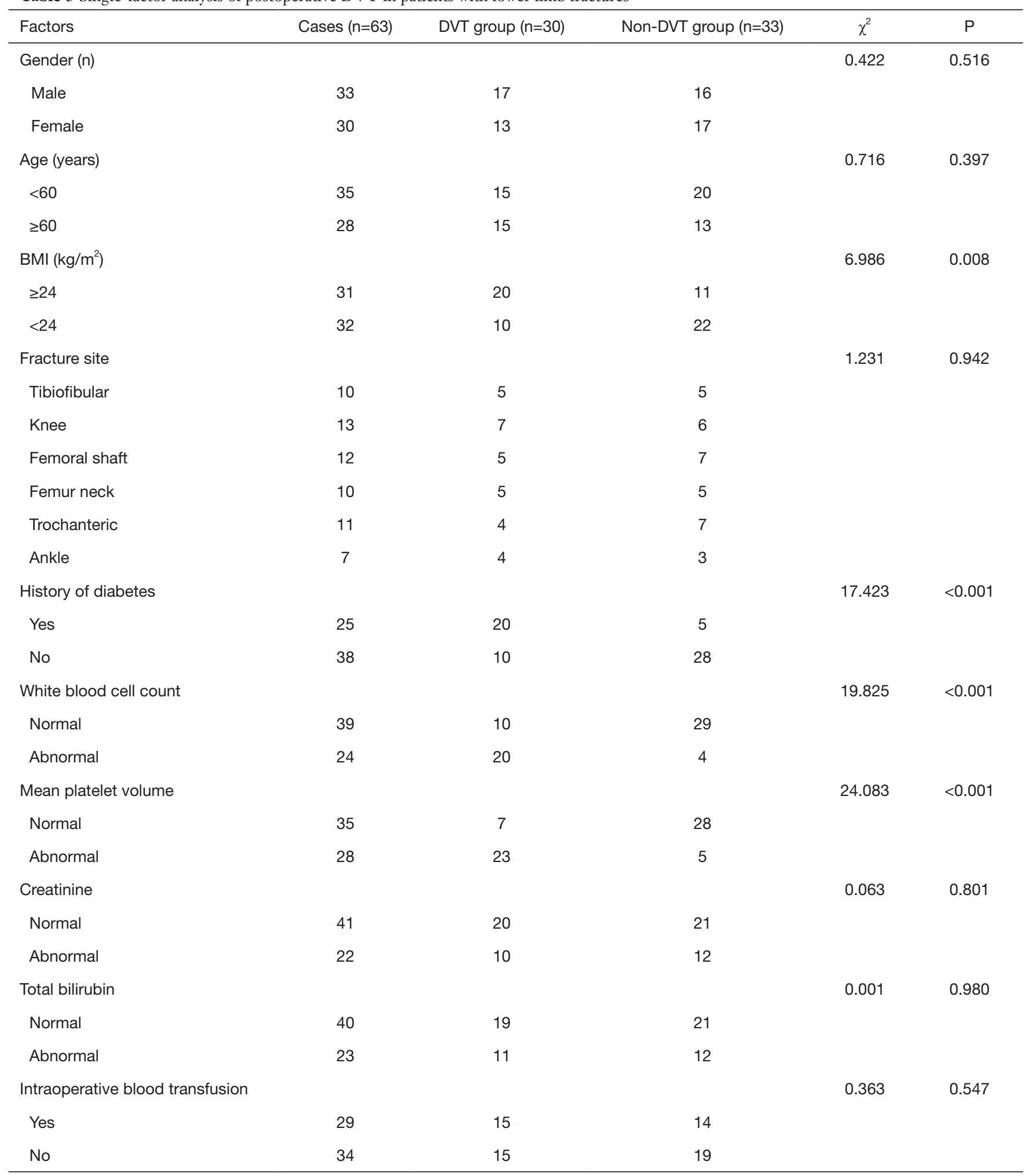

Table 3 (continued) 
Table 3 (continued)

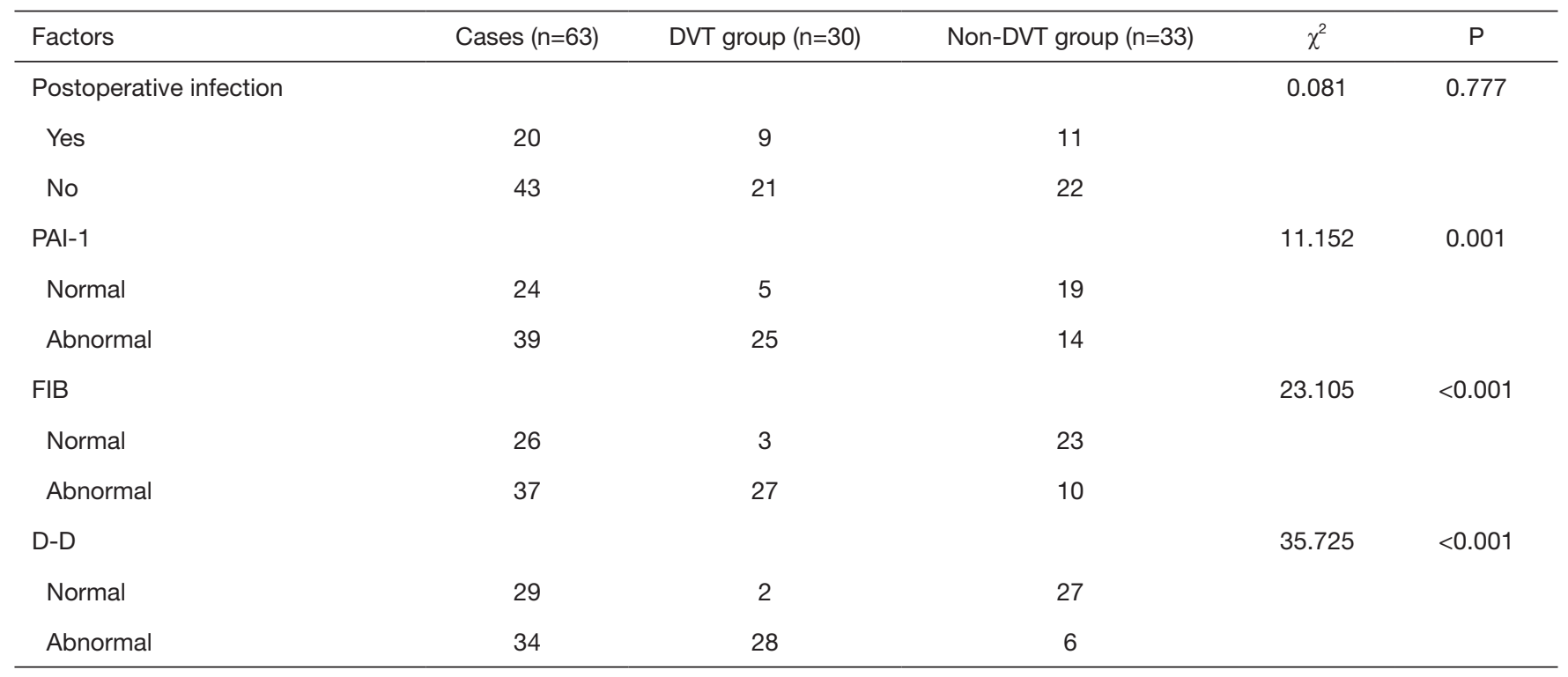

DVT, deep venous thrombosis; PAI-1, plasminogen activator inhibitor-1; FIB, fibrinogen; D-D, D-dimer.

Table 4 Multivariate analysis of recent prognosis of patients with tibial plateau fractures

\begin{tabular}{|c|c|c|c|c|c|}
\hline Factors & Regression coefficient & SEM & Wald $\chi^{2}$ & $\mathrm{P}$ & OR $(95 \% \mathrm{Cl})$ \\
\hline $\mathrm{BMI} \geq 24 \mathrm{~kg} / \mathrm{m}^{2}$ (control group: $<24 \mathrm{~kg} / \mathrm{m}^{2}$ ) & 0.795 & 0.645 & 4.539 & 0.794 & $2.22(0.63-7.86)$ \\
\hline $\begin{array}{l}\text { With a history of diabetes (control group: without a } \\
\text { history of diabetes) }\end{array}$ & 0.743 & 0.691 & 4.621 & 0.463 & $2.10(0.54-8.15)$ \\
\hline $\begin{array}{l}\text { With postoperative infections (control group: without } \\
\text { postoperative infections) }\end{array}$ & 0.851 & 0.141 & 4.792 & $<0.001$ & $2.34(1.78-3.09)$ \\
\hline $\begin{array}{l}\text { Abnormal mean platelet volume (control group: } \\
\text { normal) }\end{array}$ & 0.951 & 0.216 & 4.913 & $<0.001$ & $2.59(1.69-3.95)$ \\
\hline Abnormal PAI-1 level (control group: normal) & 0.723 & 0.191 & 4.948 & $<0.001$ & $2.07(1.42-3.00)$ \\
\hline
\end{tabular}

PAI-1, plasminogen activator inhibitor-1; FIB, fibrinogen; D-D, D-dimer.

mechanism that can maintain the permeability of the blood vessel wall, and maintain the blood flow state and tissue repair (11). When fibrin clots are formed, the plasminogen activator can convert plasminogen into plasmin and trigger the process of fibrinolysis to degrade the fibrin clots into fibrin products. Of these products, only D-D cross-linked fragments can reflect thrombolytic activity after thrombus formation. Therefore, the quantitative detection of D-D is used clinically as a sensitive indicator for the diagnosis and screening of newly-formed thrombi (12). Related research has found that the level of D-D increases along with the hyperactivity of the fibrinolytic system (13). It is believed that the balance between coagulation and the fibrinolytic system in patients could possibly be assessed by detecting the changes in the D-D level, so as to correctly evaluate the necessity of anticoagulant treatment. The results of 
this study showed that the level of D-D was significantly increased in patients with DVT following lower limb fracture surgery $(\mathrm{P}<0.05)$, which also confirms the value of the D-D level in the clinical prediction of DVT.

PAI-1 is a specific inhibitor of both tissue-type and urokinase-type plasminogen activators. When the active form of PAI-1 enters the bloodstream, it works to suppress the aforementioned plasminogen activators, thereby reducing the amount of plasminogen in the blood. This in turn inhibits fibrin degradation, and creates favorable conditions for the formation of a thrombus. Walton (14) and Wolberg (15) reported that the composition of venous thrombosis is mediated by PAI-1. In this study, we found that the level of PAI-1 in the DVT group was significantly higher than the control group $(\mathrm{P}<0.05)$ (Table 2), which is consistent with the previous reports.

FIB is a protein that is synthesized in the liver and is involved in coagulation. It acts in the body to promote platelet aggregation and the growth, proliferation, and contraction of smooth muscle and endothelial cells, increase blood viscosity and peripheral resistance, and cause endothelial cell damage (16). In addition, it can also accelerate thrombosis by promoting the synthesis of collagen, deoxyribonucleic acid (DNA), chemotactic monocytes, and macrophages to migrate to the intima. Thus, monitoring of FIB levels is clinically significant for the diagnosis of cardiovascular diseases. Our study compared the pre- and postoperative levels of D-D and FIB in lower limb fracture patients, and found that the plasma levels in patients with DVT at 1 hour postoperatively were higher than those in the non-DVT group at the same time.

ROC curves were used to analyze the predictive value of PAI-1, FIB, D-D, and the combination of these three indicators for DVT following lower limb fracture surgery. As shown in Figure 1, the area under the curve of each index was $0.792,0.429,0.966$, and 0.992 , respectively. The detection of the combination of all three indicators exhibited the best predictive value, which further confirms that the combined detection of plasma PAI- 1, D-D, and FIB levels has clinical value for the diagnosis of DVT. Further analysis of the unconditional multivariate logistic regression model showed that postoperative infection, abnormal white blood cell count, and abnormal mean platelet volume were risk factors affecting postoperative DVT in patients with lower limb fractures $(\mathrm{P}<0.05)$. This phenomenon may be related to the damage of the blood vessel wall following an infection, which can further accelerate thrombosis. Thus, attention should be given to the treatment of infection following surgery for lower limb fractures (17). Abnormal levels of PAI-1, FIB, and D-D were also factors affecting postoperative DVT in patients with lower limb fractures $(\mathrm{P}<0.05)$, which further suggests that dynamic monitoring of PAI-1, FIB, and D-D levels may be a good predictive indicator of early postoperative thrombosis. However, it should be noted that the small sample size in this study presents a limitation. Larger sample sizes are required in future research.

In conclusion, there are numerous factors affecting postoperative DVT. In this study, we found that the levels of PAI-1, FIB, and D-D were significantly increased in patients with DVT following lower limb fracture surgery. Thus, early monitoring of PAI-1, FIB, D-D levels, and coagulation function is a good predictive indicator of postoperative thrombosis.

\section{Acknowledgments}

Funding: None.

\section{Footnote}

Reporting Checklist: The authors have completed the MDAR reporting checklist. Available at http://dx.doi.org/10.21037/ apm-20-1604

Data Sharing Statement: Available at http://dx.doi. org/10.21037/apm-20-1604

Conflicts of Interest: All authors have completed the ICMJE uniform disclosure form (available at http://dx.doi. org/10.21037/apm-20-1604). The authors have no conflicts of interest to declare.

Ethical Statement: The authors are accountable for all aspects of the work in ensuring that questions related to the accuracy or integrity of any part of the work are appropriately investigated and resolved. All procedures performed in this study involving human participants were in accordance with the Declaration of Helsinki (as revised in 2013). This study was approved by Sport Hospital Affiliated to CDSU (No. 2018-12). And all the patients were signed the informed consent.

Open Access Statement: This is an Open Access article distributed in accordance with the Creative Commons Attribution-NonCommercial-NoDerivs 4.0 International 
License (CC BY-NC-ND 4.0), which permits the noncommercial replication and distribution of the article with the strict proviso that no changes or edits are made and the original work is properly cited (including links to both the formal publication through the relevant DOI and the license). See: https://creativecommons.org/licenses/by-nc-nd/4.0/.

\section{References}

1. Ohashi R, Sugimura M, Kawamura T, et al. Sensitivity to activated protein $\mathrm{C}$ in patients with deep vein thrombosis during early puerperium period. Acta Obstet Gynecol Scand 2005;84:799-801.

2. Cosmi B, Palareti G. "Early thrombus removal" in iliacfemoral deep vein thrombosis for prevention of postthrombotic syndrome. Ann Transl Med 2019;7:S343.

3. Ikeda T, Miyamoto H, Hashimoto K, et al. Predictable factors of deep venous thrombosis in patients undergoing spine surgery. J Orthop Sci 2017;22:197-200.

4. Cini M, Legnani C, Frascaro M, et al. D-dimer use for deep venous thrombosis exclusion in elderly patients: a comparative analysis of three different approaches to establish cut-off values for an assay with results expressed in D-dimer units. Int J Lab Hematol 2014;36:541-7.

5. Chen S, Gao Y, Wang W, et al. Long-term clinical outcomes of complicated retrievable inferior vena cava filter for deep venous thrombosis patients: safety and effectiveness. Med Sci Monit 2019;25:128-34.

6. Tala JA, Polikoff LA, Pinto MG, et al. Protein biomarkers for incident deep venous thrombosis in critically ill adolescents: An exploratory study. Pediatr Blood Cancer 2020;67:e28159.

7. Vuckovic BA, Djeric MJ, Tomic BV, et al. Influence of decreased fibrinolytic activity and plasminogen activator inhibitor-1 4G/5G polymorphism on the risk of venous thrombosis. Blood Coagul Fibrinolysis 2018;29:19-24.

Cite this article as: Cheng J, Fu Z, Zhu J, Zhou L, Song W. The predictive value of plasminogen activator inhibitor-1, fibrinogen, and D-dimer for deep venous thrombosis following surgery for traumatic lower limb fracture. Ann Palliat Med 2020;9(5):3385-3392. doi: 10.21037/apm-20-1604
8. Palareti G. D-Dimer Testing in patients with a first unprovoked venous thromboembolism. Ann Intern Med 2015;162:670-1.

9. Tsantarliotou MP, Lavrentiadou SN, Psalla DA, et al. Suppression of plasminogen activator inhibitor-1 (PAI-1) activity by crocin ameliorates lipopolysaccharide-induced thrombosis in rats. Food Chem Toxicol 2019;125:190-7.

10. Cao Z, Dong Y, Zeng J, et al. Whole-exome sequencing identified novel mutations in FGA and FGG genes in the patients with decreased fibrinogen. Thromb Res 2019;177:79-82.

11. Cai HX, Li XQ, Wang SF. Prognostic value of fibrinogen and D-dimer-fibrinogen ratio in resectable gastrointestinal stromal tumors. World J Gastroenterol 2018;24:5046-56.

12. Cosmi B, Palareti G. Update on the predictive value of $\mathrm{D}$-dimer in patients with idiopathic venous thromboembolism. Thromb Res 2010;125 Suppl 2:S62-5.

13. Huebner BR, Moore EE, Moore HB, et al. Thrombin stimulates increased plasminogen activator inhibitor-1 release from liver compared to lung endothelium. J Surg Res 2018;225:1-5.

14. Walton BL, Byrnes JR, Wolberg AS. Fibrinogen, red blood cells, and factor XIII in venous thrombosis. J Thromb Haemost. 2015;13 Suppl 1:S208-15.

15. Wolberg AS. Fibrinogen and factor XIII: newly recognized roles in venous thrombus formation and composition. Curr Opin Hematol 2018;25:358-64.

16. Wilbur J, Shian B. Deep venous thrombosis and pulmonary embolism: current therapy. Am Fam Physician 2017;95:295-302.

17. Jiang K, Li XQ, Sang HF, et al. Mid-term outcome of endovascular treatment for acute lower extremity deep venous thrombosis. Phlebology 2017;32:200-6.

(English Language Editor: A. Kassem) 